\title{
Exchange Functionals and Basis Set Comparisons for Theoretical Studies of $\mathrm{ZnO}$ nonoclusters
}

\author{
Duwage C. Perera ${ }^{\mathrm{a}}$ and Jayendran C. Rasaiah ${ }^{\mathrm{a}, 1}$ \\ ${ }^{a}$ Department of Chemistry, University of Maine, Orono, ME 04469 \\ This manuscript was compiled on May 13, 2021
}

\begin{abstract}
Catalysts made of nano-scaled metal oxide clusters can push the limits of chemical reactions in the manufacture of paints, cosmetics, and pharmaceuticals. The $\mathrm{ZnO}$ clusters can also act as semiconductors with a wide band gap of $3.4 \mathrm{eV}$ at $300 \mathrm{~K}$, and are prospective photocatalysts in many reactions including $\mathrm{H}_{2}$ production in water splitting reactions. In this project, we studied the structural (geometry) and electronic properties (vertical detachment energy and electron affinity) of $\mathrm{ZnO}$ monomers and dimers that form model $\mathrm{ZnO}$ clusters, using density functional theory (DFT) with many different exchange functionals and 29 basis sets to optimize their choice. We compared the singlet-triplet energy gaps of small $\mathrm{ZnO}$ clusters to find the optimal ZnO cluster size and the best theoretical method to investigate their photocatalytic water splitting activity.

Our results show that B3LYP/DGDZVP2 level of exchange functional/basis set theory is the most efficient and fastest of the ones considered. Comparison of the singlet-triplet energy gaps shows that the trimer $(\mathrm{ZnO})_{3}$, with an energy gap of $58.66 \mathrm{k} \mathrm{cal} / \mathrm{mol}$, is approximately equal to the energy of a visible photon at $555 \mathrm{~nm}$ and a HOMO-LUMO gap of $4.4 \mathrm{eV}$, and is the best choice amongst the $(\mathrm{ZnO})_{n}$ clusters of different sizes when the number of monomers $\mathrm{n}$ in the clusters ranges from 1 to 6 . We used the Gaussian 16 software package for all the calculations.
\end{abstract}

Zinc Oxide Clusters | Density Functional Theory | Basis Set Comparison |Exchange functional | Vertical Detachment Energy | Electron Affinity etal oxides have been used in many ways over the past decades. Depending on the metal, the oxides can be utilized as catalysts, sensors, in food industry, cosmetics, medicine and solar cells. Scaling down from macro to nano, both the physical and chemical properties of each metal oxide are changed, and nano scaled metal oxide clusters can become promising material to push the limits of their applications (1$5)$. Accordingly, there has been a growing interest in studying the properties of metal oxide nanoclusters using both theoretical and experimental methods (6-9). Zinc oxide $(\mathrm{ZnO})$ is such a material with potential applications of interest in many industries including paint, cosmetics, pharmaceuticals and catalysis (10-19). ZnO nanoparticles also have been identified as potential adsorbents of organic dyes, gasses and heavy metals $(20,21)$. It is a semiconductor with a wide band gap of 3.4 $\mathrm{eV}$ at $300 \mathrm{~K}$ and has unique magnetic, optical and electronic properties (22-24). Moreover those properties can change considerably when the particle dimension and size change to the nanoscale. $\mathrm{ZnO}$ nanoparticles with different morphologies including nanorods, nanosheets, nanowires and nanoflowers can be synthesized using thermal decomposition, the sol-gel method, forced hydrolysis and hydro-thermal method $(25,26)$. have been studied both experimentally and theoretically.
Bovhyra et al. used density functional theory (DFT) to study the structural and electronic properties of $(\mathrm{ZnO})_{\mathrm{n}}$ $(\mathrm{n}=34,60)$ nanoclusters (27). From their results, the most stable $(\mathrm{ZnO})_{34}$ nanoclusters were fullerene - like hollow structures and the most energetically favorable $(\mathrm{ZnO})_{60}$ had a sodalite - type structure made with seven $(\mathrm{ZnO})_{12}$ clusters with common quadrangle edges. The energy range of the highest occupied molecular orbital (HOMO) and the lowest unoccupied molecular orbital (LUMO) for those clusters are $1.93-2.40 \mathrm{eV}$. A study by Woodley et al. reported the stable and low energy meta-stable structures of $(\mathrm{ZnO})_{n},(n=1-32)$ clusters using evolutionary algorithm techniques (28). Their findings include, $2 \mathrm{D}$ rings for $\mathrm{n}=2-7$ that are less than $0.8 \mathrm{~nm}$ in diameter and spheroid bubbles upto $n=32$ that are less than $1.2 \mathrm{~nm}$ in diameter.

Another theoretical study by Szakacs et.al shows the relative thermodynamic stability of two different $\mathrm{ZnO}$ nanocluster shapes; needles and plates. The electronic structure calculations of $\mathrm{ZnO}$ needles and plates nanoclusters shows, the $\mathrm{Zn}_{\mathrm{i}} \mathrm{O}_{\mathrm{i}}$; where $\mathrm{i}=6,9,12,15$ and 18 , needles have higher stability that increased with the number of stacked rings. They also studied $\mathrm{Zn}_{3} \mathrm{O}_{3}$ as the most successful building block to form larger clusters rather than adding individual or pairs of $\mathrm{Zn}$ and $\mathrm{O}$ atoms (29).

An extensive theoretical study on the structural and electronic properties of $(\mathrm{ZnO})_{\mathrm{n}}, \mathrm{n} \leq 168$ has been carried out by Chen et al (30). Their major findings include the presence of new magic numbers for $(\mathrm{ZnO})_{n}$ for structures at $\mathrm{n}=78,100,132$ and 168 with the particle stability increasing for structures with single to double and triple layered octahedral cage morphologies. The higher stability in multilayered particles is due to the lack of terminal surface atoms and the effective interlayer stacking of hexagonal cells. Chen et al. have also summarized the stability of small $\mathrm{ZnO}$ clusters. The lowest energy isomers for $(\mathrm{ZnO})_{\mathrm{n}} \mathrm{n}=3-5$ are predicted to be ringlike 2 - dimensional (2D) structures that have higher stability compared to the open and $3 \mathrm{D}$ structures .

To fill the gap in information lacking in previous investigations of anionic $\mathrm{ZnO}$ clusters, Castlemen Jr et al. investigated the relative stabilities and electronic properties of small $\mathrm{ZnO}$ clusters using density functional theory (DFT) with both B3LYP and PBEPBE functionala and 6-311++G(3d), augcc-pvQZ and LanL2DZ basis sets (31), and have compared their calculations with published experimental results. This combined experimental and theoretical study reported the

\section{The authors declare no competing financial interest}

${ }^{1}$ To whom correspondence should be addressed. E-mail: rasaiah@maine.edu 
mass spectrum of anionic $\mathrm{Zn}_{n} \mathrm{O}_{m}(\mathrm{n}=1-6$ and $\mathrm{m}=1-7)$ clusters and predicted the ground state geometries and stabilities of $\mathrm{Zn}_{3} \mathrm{O}_{\mathrm{m}}$ and $\mathrm{Zn}_{3} \mathrm{O}_{\mathrm{m}}{ }^{-}(\mathrm{m}=3-5)$.

In this paper, we set the stage for further investigations of the photocatalytic properties of $\mathrm{ZnO}$ clusters by examining the structural and electronic properties of the monomer and dimer of $\mathrm{ZnO}$ using different functionals and basis sets to investigate improved methods of property analysis. Moreover, we calculate the energy gap of singlet and triplet states of small $\mathrm{ZnO}$ clusters and HOMO-LUMO energy gaps with selected basis sets as a first step towards understanding the photocatalytic properties of $\mathrm{ZnO}$ clusters, and specifically as a first step towards the study of $\mathrm{H}_{2}$ and $\mathrm{O}_{2}$ production by water splitting reaction. To the best of our knowledge, this has not been previously reported theoretically for $\mathrm{ZnO}$ clusters.

\section{Computational Method}

We first chose the monomer and the dimer of $\mathrm{ZnO}$ to study their structural and electronic properties using different functionals in DFT. We chose 30 different basis sets by using standard and augmented Dunning and Pople style bases: $3-21 \mathrm{G}, 6-31 \mathrm{G}, 6-31+\mathrm{G}, 6-31++\mathrm{G}, 6-311 \mathrm{G}, 6-$ $311++\mathrm{G}, 6-31 \mathrm{G}(\mathrm{d}), 6-31 \mathrm{G}(\mathrm{d}, \mathrm{p}), 6-31+\mathrm{G}(\mathrm{d}), 6-31+\mathrm{G}(2 \mathrm{~d}), 6-$ $31+\mathrm{G}(2 \mathrm{~d}, \mathrm{p}), 6-31+\mathrm{G}(2 \mathrm{df}, \mathrm{p}), 6-31+\mathrm{G}(2 \mathrm{df}, 2 \mathrm{p}), 6-31+\mathrm{G}(3 \mathrm{df}, 2 \mathrm{p})$, 6-31++G(d), 6-31++G(d,p), 6-311+G(d), 6-311G(d,p), 6$311++\mathrm{G}(\mathrm{d}), 6-311++\mathrm{G}(\mathrm{d}, \mathrm{p})$, cc-PVQZ, aug-cc-PVDZ, aug-ccPVTZ, DGDZVP, DGDZVP2, Def2TZVP, LANL2DZ, CEP$121 \mathrm{G}, \mathrm{TZVP}, \mathrm{QZVP}$.

First we optimized the $\mathrm{ZnO}$ monomer and dimer structures using B3LYP, B3PW91, PBEPBE, BVP86, BP86, M06 exchange functionals in DFT and the $\operatorname{CCSD}(\mathrm{T})$ method with 30 different basis sets. We analyzed bond lengths, bond angles and mulliken charges on atoms as structural properties and the vertical detachment energy (VDE) and electron affinity (EA) as electronic properties of each optimized structure. Additionally we compared the HOMO-LUMO gap, the difference between singlet and triplet energies and infrared spectra of $(\mathrm{ZnO}) \mathrm{n}$ clusters, with $\mathrm{n}=1-6$, to evaluate candidates with suitable photocatalytic properties. We compared our calculated values with previously published experimental results (31) to identify the best basis set candidates.

All geometry optimization and structural and electronic property calculations were carried out using the Gaussian 16 software package (32) and GaussView 6 graphical interface (33).

\section{Results and Discussion}

A. Comparison of Structural Properties. Geometry optimization is the process of finding the positions of nuclei for which the potential energy is minimized. We optimized the $\mathrm{ZnO}$ monomer and dimer and compared the structural properties using the different DFT and $\operatorname{CCSD}(\mathrm{T})$ method with 30 different basis sets. Figure 1 illustrates the optimized monomer (a) and dimer (b) structures of $\mathrm{ZnO}$ using DFT with B3LYP/DGDZVP2 combination of exchange functional and basis set. We selected the bond lengths (2Zn - 10 for both monomer and dimer as shown in the Figure 1), and dimer bond angles (2Zn-1O-3Zn and 1O-2Zn-4O) and mulliken charges on each atom (labeled as in the Figure 1) as characteristic structural properties.
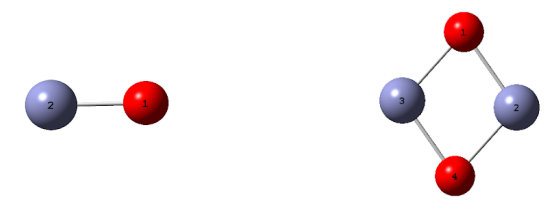

(a) ZnO Monomer

(b) ZnO Dimer

Fig. 1. Optimized (a) monomer and (b) dimer structures at B3LYP/ DGDZVP2 level of theory. $\mathrm{Zn}$ and $\mathrm{O}$ atoms are represented by light blue and red colors respectively.

Table 1. Monomer Bond Length (Zn-0)/Å

\begin{tabular}{|c|c|c|c|c|c|c|c|c|}
\hline \multicolumn{2}{|c|}{ Functional } & \multicolumn{6}{|c|}{ DFT } & \multirow{2}{*}{$\operatorname{CcsD}(T)$} \\
\hline & & B3LYP & B3PW91 & PBEPBE & BVP86 & BP86 & M06 & \\
\hline 1 & $3-21 G$ & 1.609 & 1.607 & 1.603 & 1.603 & 1.603 & 1.602 & 1.658 \\
\hline 2 & $6-31 G$ & 1.695 & 1.687 & 1.684 & 1.683 & 1.683 & 1.685 & 1.748 \\
\hline 3 & $6-31+G$ & 1.740 & 1.727 & 1.731 & 1.729 & 1.730 & 1.730 & 1.780 \\
\hline 4 & $6-31++G$ & 1.740 & 1.727 & 1.731 & 1.729 & 1.730 & 1.730 & 1.780 \\
\hline 5 & $6-311 G$ & 1.818 & 1.799 & 1.791 & 1.791 & 1.792 & 1.822 & 1.829 \\
\hline 6 & $6-311++G$ & 1.740 & 1.728 & 1.731 & 1.730 & 1.730 & 1.730 & 1.768 \\
\hline 7 & $6-31 G(d)$ & 1.670 & 1.663 & 1.661 & 1.660 & 1.661 & 1.658 & 1.700 \\
\hline 8 & $6-31 G(d, p)$ & 1.670 & 1.663 & 1.661 & 1.660 & 1.661 & 1.658 & 1.700 \\
\hline 9 & $6-31+G(d)$ & 1.712 & 1.700 & 1.705 & 1.704 & 1.705 & 1.699 & 1.730 \\
\hline 10 & $6-31+G(2 d)$ & 1.712 & 1.700 & 1.705 & 1.704 & 1.705 & 1.700 & 1.729 \\
\hline 11 & $6-31+G(2 d, p)$ & 1.712 & 1.700 & 1.705 & 1.704 & 1.705 & 1.700 & 1.729 \\
\hline 12 & $6-31+G(2 d f, p)$ & 1.710 & 1.698 & 1.703 & 1.702 & 1.703 & 1.698 & 1.718 \\
\hline 13 & $6-31+G(2 d f, 2 p)$ & 1.710 & 1.698 & 1.703 & 1.702 & 1.703 & 1.698 & 1.718 \\
\hline 14 & $6-31+G(3 d f, 2 p)$ & 1.708 & 1.696 & 1.701 & 1.700 & 1.701 & 1.695 & 1.715 \\
\hline 15 & $6-31++G(d)$ & 1.712 & 1.700 & 1.705 & 1.704 & 1.705 & 1.699 & 1.730 \\
\hline 16 & $6-31++G(d, p)$ & 1.712 & 1.700 & 1.705 & 1.704 & 1.705 & 1.699 & 1.730 \\
\hline 17 & $6-311+G(d)$ & 1.720 & 1.709 & 1.713 & 1.712 & 1.713 & 1.709 & 1.731 \\
\hline 18 & 6-311G(d,p) & 1.791 & 1.773 & 1.766 & 1.766 & 1.767 & 1.793 & 1.788 \\
\hline 19 & $6-311++G(d)$ & 1.720 & 1.709 & 1.713 & 1.712 & 1.713 & 1.709 & 1.731 \\
\hline 20 & $6-311++G(d, p)$ & 1.720 & 1.709 & 1.713 & 1.712 & 1.713 & 1.709 & 1.731 \\
\hline 21 & cc-PVQZ & 1.707 & 1.696 & 1.700 & 1.699 & 1.700 & 1.694 & 1.712 \\
\hline 22 & aug-cc-PVDZ & 1.712 & 1.701 & 1.705 & 1.704 & 1.705 & 1.699 & 1.714 \\
\hline 23 & aug-cc-PVTZ & 1.710 & 1.699 & 1.703 & 1.702 & 1.703 & 1.696 & 1.719 \\
\hline 24 & DGDZVP & 1.726 & 1.713 & 1.715 & 1.715 & 1.716 & 1.714 & 1.755 \\
\hline 25 & DGDZVP2 & 1.718 & 1.706 & 1.708 & 1.707 & 1.707 & 1.706 & 1.746 \\
\hline 26 & Def2TZVP & 1.713 & 1.701 & 1.705 & 1.705 & 1.705 & 1.702 & 1.717 \\
\hline 27 & LANL2DZ & 1.764 & 1.754 & 1.765 & 1.764 & 1.765 & 1.753 & 1.783 \\
\hline 28 & CEP-121G & 1.732 & 1.719 & 1.720 & 1.719 & 1.720 & 1.718 & 1.757 \\
\hline 29 & TZVP & 1.722 & 1.709 & 1.711 & 1.710 & 1.711 & 1.711 & 1.740 \\
\hline 30 & QZVP & 1.709 & 1.697 & 1.702 & 1.701 & 1.702 & 1.696 & 1.714 \\
\hline
\end{tabular}

Table 2. Dimer Bond Length (Zn-O)/Å

\begin{tabular}{|c|c|c|c|c|c|c|c|c|}
\hline & \multirow{2}{*}{$\begin{array}{l}\text { Functional } \\
\text { Basis Set }\end{array}$} & \multicolumn{6}{|c|}{ DFT } & \multirow{2}{*}{$\operatorname{CcsD}(T)$} \\
\hline & & B3LYP & B3PW91 & PBEPBE & BVP86 & BP86 & M06 & \\
\hline 1 & $3-21 G$ & 1.825 & 1.822 & 1.832 & 1.831 & 1.832 & 1.813 & 1.858 \\
\hline 2 & $6-31 G$ & 1.891 & 1.882 & 1.901 & 1.900 & 1.900 & 1.874 & 1.925 \\
\hline 3 & $6-31+G$ & 1.921 & 1.908 & 1.936 & 1.934 & 1.935 & 1.901 & 1.946 \\
\hline 4 & $6-31++G$ & 1.921 & 1.908 & 1.936 & 1.934 & 1.935 & 1.901 & 1.946 \\
\hline 5 & 6-311G & 1.925 & 1.909 & 1.942 & 1.940 & 1.941 & 1.900 & - \\
\hline 6 & $6-311++G$ & 1.915 & 1.904 & 1.931 & 1.928 & 1.929 & 1.896 & 1.932 \\
\hline 7 & $6-31 G(d)$ & 1.856 & 1.847 & 1.863 & 1.862 & 1.863 & 1.841 & 1.868 \\
\hline 8 & $6-31 G(d, p)$ & 1.856 & 1.847 & 1.863 & 1.862 & 1.863 & 1.841 & 1.868 \\
\hline 9 & $6-31+G(d)$ & 1.890 & 1.879 & 1.902 & 1.901 & 1.902 & 1.873 & 1.898 \\
\hline 10 & $6-31+G(2 d)$ & 1.887 & 1.876 & 1.900 & 1.899 & 1.899 & 1.870 & 1.894 \\
\hline 11 & $6-31+G(2 d, p)$ & 1.887 & 1.876 & 1.900 & 1.899 & 1.899 & 1.870 & 1.894 \\
\hline 12 & $6-31+G(2 d f, p)$ & 1.886 & 1.875 & 1.899 & 1.898 & 1.899 & 1.869 & 1.884 \\
\hline 13 & $6-31+G(2 d f, 2 p)$ & 1.886 & 1.875 & 1.899 & 1.898 & 1.899 & 1.869 & 1.884 \\
\hline 14 & $6-31+G(3 d f, 2 p)$ & 1.884 & 1.872 & 1.896 & 1.895 & 1.896 & 1.867 & - \\
\hline 15 & $6-31++G(d)$ & 1.890 & 1.879 & 1.902 & 1.901 & 1.902 & 1.873 & 1.898 \\
\hline 16 & $6-31++G(d, p)$ & 1.890 & 1.879 & 1.902 & 1.901 & 1.902 & 1.873 & 1.898 \\
\hline 17 & $6-311+G(d)$ & 1.898 & 1.888 & 1.913 & 1.911 & 1.912 & 1.881 & 1.897 \\
\hline 18 & 6-311G(d,p) & 1.899 & 1.884 & 1.915 & 1.913 & 1.914 & 1.876 & - \\
\hline 19 & $6-311++G(d)$ & 1.898 & 1.888 & 1.913 & 1.911 & 1.912 & 1.881 & 1.897 \\
\hline 20 & $6-311++G(d, p)$ & 1.898 & 1.888 & 1.913 & 1.911 & 1.912 & 1.881 & 1.897 \\
\hline 21 & aug-cc-PVDZ & 1.888 & 1.879 & 1.901 & 1.899 & 1.900 & 1.870 & 1.892 \\
\hline 22 & aug-cc-PVTZ & 1.886 & 1.876 & 1.899 & 1.897 & 1.897 & 1.867 & - \\
\hline 23 & DGDZVP & 1.907 & 1.896 & 1.922 & 1.921 & 1.922 & 1.886 & 1.922 \\
\hline 24 & DGDZVP2 & 1.900 & 1.890 & 1.913 & 1.911 & 1.912 & 1.882 & 1.917 \\
\hline 25 & Def2TZVP & 1.891 & 1.881 & 1.905 & 1.904 & 1.904 & 1.873 & 1.884 \\
\hline 26 & LANL2DZ & 1.932 & 1.923 & 1.953 & 1.951 & 1.952 & 1.916 & 1.948 \\
\hline 27 & CEP-121G & 1.908 & 1.897 & 1.921 & 1.920 & 1.921 & 1.892 & 1.922 \\
\hline 28 & TZVP & 1.898 & 1.888 & 1.913 & 1.911 & 1.912 & 1.881 & 1.902 \\
\hline 29 & QZVP & 1.885 & 1.875 & 1.897 & 1.896 & 1.897 & 1.867 & 1.876 \\
\hline
\end{tabular}

The monomer and dimer bond lengths calculated using 30 
different basis sets and 6 different DFT functionals and $\operatorname{CCSD}(\mathrm{T})$ method are shown in the Tables 1 and 2 respectively. All other results are tabulated in the supporting information (SI) document.

We compared the bond lengths of both monomer and dimer with the published experimental values (the bond length of $\mathrm{Zn}-\mathrm{O}$ is $1.719 \AA$ and 1.787 for its anion. The bond lengths, calculated using B3LYP functional vary widely from $1.609 \AA-1.818 \AA$. However, basis sets $6-311+\mathrm{G}(\mathrm{d})$, 6-311++G(d),6-311++G(d,p) and DGDZVP2 show bond lemgths in good agreement with the experimental value within \pm 0.01 difference. Monomer bond lengths calculated using the B3LYP functional are comparably closer to experiment to within the first decimal using the $\operatorname{CCSD}(\mathrm{T})$ method. The $\mathrm{Zn}-\mathrm{O}$ bond length in the dimer is longer than in the monomer.

Dimer bond angles, 2Zn-1O-3Zn and 1O-2Zn-4O are shown in Figure 1(b) and presented in Table 3. The 2Zn-1O-3Zn angle is between $73.17^{\circ}-79.59^{\circ}$, while the $10-2 \mathrm{Zn}-4 \mathrm{O}$ angles, which are larger, are between $100.06^{\circ}-106.86^{\circ}$. In comparison, a theoretical study by Ugalde et.al on the stability of global minimum for small $\mathrm{ZnO}$ clusters shows the $1 \mathrm{O}-2 \mathrm{Zn}-4 \mathrm{O}$ angle is $103.7^{\circ}$ and the $\mathrm{Zn}-\mathrm{O}$ bond length is $1.892 \AA$ for the $\mathrm{ZnO}$ dimer using B3LYP functional and the relativistic compact effective core potentials and shared-exponent basis set of Stevens, Krauss, Basch and Jasien (SKBJ), in good agreement with our results. (34)

B. Comparison of Electronic Properties. Amongst the electronic properties, we compare the expermental $(35,36)$ electron affinities (EA) and vertical detachment energies (VDE) of both $\mathrm{ZnO}$ monomer and dimer with our calculated values using equation 1 and 2 (37).

Electron Affinity (EA) $=\mathrm{E}($ optimized neutral molecule)E(optimized anion) [1]

Vertical Detachment Energy $(\mathrm{VDE})=\mathrm{E}$ (neutral at optimized anion geometry) $-\mathbf{E}$ (optimized anion) $[2]$

The difference in energy between the neutral molecule and the negative ion of the molecule in their ground state is the $\mathrm{EA}$ of a molecule. Positive $\mathrm{EA}$ values indicate thermodynamically stable negative ions. All the EA values for the monomer recorded in the Table 4 are positive except for the $\operatorname{CCSD}(\mathrm{T}) / 6-311 \mathrm{G}(\mathrm{d}, \mathrm{p})$ and $\operatorname{CCSD}(\mathrm{T}) /$ aug-cc-PVDZ caculations. The positive EA values for the monomer are in the range of $0.567-2.538 \mathrm{eV}$. According to an experimental study by Fancher et.al and Kim et.al using photoelectron spectra of $\mathrm{ZnO}$ and $\mathrm{ZnO}^{-}$, the EA of the monomer $\mathrm{ZnO}$ is $2.088 \pm 0.010 \mathrm{eV}(35,36)$. From Table 4, $6-31+\mathrm{G}, 6-31++\mathrm{G}, 6-31+\mathrm{G}(3 \mathrm{df}, 2 \mathrm{p})$ and aug-cc-PVTZ basis sets from $\operatorname{CCSD}(\mathrm{T})$ method we see that they agree with the experimental value. Comparing the DFT results, from B3LYP functional DGDZVP2 and CEP-121G; B3PW91 functional LANL2DZ; PBEPBE functional cc-PVQZ and aug-cc-PVDZ; and M06 functional cc-PVQC basis sets are also in accord with the experimental value.
Table 3. Dimer Bond Angle (2Zn-10-3Zn) $/{ }^{\circ}$ in black and Dimer Bond Angle $(10-2 \mathrm{Zn}-40) /{ }^{\circ}$ in orange

\begin{tabular}{|c|c|c|c|c|c|c|c|c|}
\hline & \multirow{2}{*}{$\begin{array}{l}\text { Functional } \\
\text { Basis Set }\end{array}$} & \multicolumn{6}{|c|}{ DFT } & \multirow[b]{2}{*}{$\operatorname{ccsD}(T)$} \\
\hline & & B3LYP & B3PW91 & PBEPBE & BVP86 & BP86 & M06 & \\
\hline \multirow{2}{*}{1} & \multirow{2}{*}{$3-21 G$} & 74.17 & 74.31 & 73.27 & 73.17 & 73.14 & 74.62 & 75.05 \\
\hline & & 105.83 & 105.69 & 106.73 & 106.83 & 106.86 & 105.38 & 104.96 \\
\hline \multirow{2}{*}{2} & \multirow{2}{*}{$6-31 G$} & 74.87 & 74.75 & 73.26 & 73.22 & 73.20 & 74.91 & 75.06 \\
\hline & & 105.13 & 105.25 & 106.74 & 106.78 & 106.80 & 105.09 & 104.94 \\
\hline \multirow{2}{*}{3} & \multirow{2}{*}{$6-31+G$} & 76.89 & 76.49 & 75.59 & 75.65 & 75.63 & 76.79 & 75.97 \\
\hline & & 103.11 & 103.51 & 104.41 & 104.35 & 104.37 & 103.21 & 104.05 \\
\hline \multirow{2}{*}{4} & \multirow{2}{*}{$6-31++G$} & 76.89 & 76.49 & 75.59 & 75.65 & 75.63 & 76.79 & 75.97 \\
\hline & & 103.11 & 103.51 & 104.41 & 104.35 & 104.37 & 103.21 & 104.05 \\
\hline \multirow{2}{*}{5} & \multirow{2}{*}{$6-311 G$} & 77.13 & 77.25 & 76.09 & 76.06 & 76.05 & 77.04 & - \\
\hline & & 102.87 & 102.75 & 103.91 & 103.94 & 103.95 & 102.96 & \\
\hline \multirow{2}{*}{6} & & 77.20 & 76.85 & 75.83 & 75.90 & 75.88 & 76.99 & 76.67 \\
\hline & $6-311++G$ & 102.80 & 103.15 & 104.17 & 104.10 & 104.12 & 103.01 & 103.37 \\
\hline & & 74.94 & 74.81 & 73.50 & 73.49 & 73.47 & 74.97 & 74.97 \\
\hline 7 & $6-31 G(\mathrm{~d})$ & 105.06 & 105.19 & 106.50 & 106.51 & 106.53 & 105.03 & 105.03 \\
\hline & & 74.94 & 74.81 & 73.50 & 73.49 & 73.47 & 74.97 & 74.97 \\
\hline 8 & $6-31 G(d, p)$ & 105.06 & 105.19 & 106.50 & 106.51 & 106.53 & 105.03 & 105.03 \\
\hline & & 77.17 & 76.80 & 75.92 & 75.98 & 75.94 & 76.91 & 76.40 \\
\hline 9 & $6-31+G(d)$ & 102.83 & 103.20 & 104.08 & 104.02 & 104.06 & 103.09 & 103.60 \\
\hline & & 77.13 & 76.70 & 75.83 & 75.90 & 75.89 & 76.92 & 76.27 \\
\hline 10 & $6-31+G(2 d)$ & 102.87 & 103.30 & 104.17 & 104.10 & 104.11 & 103.08 & 103.72 \\
\hline & & 77.13 & 76.70 & 75.83 & 75.90 & 75.89 & 76.92 & 76.27 \\
\hline 11 & $6-31+G(2 a, p)$ & 102.87 & 103.30 & 104.17 & 104.10 & 104.11 & 103.08 & 103.72 \\
\hline & & 77.11 & 76.68 & 75.80 & 75.87 & 75.86 & 76.90 & 76.36 \\
\hline 12 & $6-31+G(2 d f, p)$ & 102.89 & 103.32 & 104.20 & 104.13 & 104.14 & 103.10 & 103.64 \\
\hline & & 77.11 & 76.68 & 75.80 & 75.87 & 75.86 & 76.90 & 76.35 \\
\hline 13 & $6-31+G(2 \mathrm{dt}, 2 \mathrm{p})$ & 102.89 & 103.32 & 104.20 & 104.13 & 104.14 & 103.10 & 103.65 \\
\hline & & 76.86 & 76.47 & 75.57 & 75.63 & 75.61 & 76.62 & - \\
\hline 14 & $6-31+G(3 d t, 2 p)$ & 103.14 & 103.53 & 104.43 & 104.37 & 104.39 & 103.38 & \\
\hline & & 77.17 & 76.80 & 75.92 & 75.98 & 75.94 & 76.91 & 76.40 \\
\hline 15 & $6-3$ & 102.83 & 103.20 & 104.08 & 104.02 & 104.06 & 103.09 & 103.60 \\
\hline & & 77.17 & 76.80 & 75.92 & 75.98 & 75.94 & 76.91 & 76.40 \\
\hline 16 & $6-31++G(d, p)$ & 102.83 & 103.20 & 104.08 & 104.02 & 104.06 & 103.09 & 103.60 \\
\hline & & 77.40 & 77.09 & 76.11 & 76.16 & 76.14 & 77.11 & 76.97 \\
\hline 17 & $6-311+G(d)$ & 102.60 & 102.91 & 103.89 & 103.84 & 103.86 & 102.89 & 103.03 \\
\hline & & 76.82 & 77.00 & 75.87 & 75.85 & 75.83 & 76.78 & - \\
\hline 18 & $6-311 G(d, p)$ & 103.18 & 103.00 & 104.13 & 104.15 & 104.17 & 103.22 & \\
\hline & & 77.40 & 77.09 & 76.11 & 76.16 & 76.14 & 77.11 & 76.97 \\
\hline 19 & & 102.60 & 102.91 & 103.89 & 103.84 & 103.86 & 102.89 & 103.03 \\
\hline & & 77.40 & 77.09 & 76.11 & 76.16 & 76.14 & 77.11 & 76.97 \\
\hline 20 & $6-311++G(d, p)$ & 102.60 & 102.91 & 103.89 & 103.84 & 103.86 & 102.89 & 103.03 \\
\hline & & 77.28 & 76.90 & 76.00 & 76.05 & 76.04 & 76.90 & 76.51 \\
\hline 21 & aug-CC-PVDZ & 102.72 & 103.10 & 104.00 & 103.95 & 103.96 & 103.10 & 103.49 \\
\hline & & 77.31 & 76.93 & 76.04 & 76.08 & 76.08 & 76.84 & - \\
\hline 22 & aug-cC-PVTZ & 102.68 & 103.07 & 103.96 & 103.92 & 103.92 & 103.16 & \\
\hline & & 77.04 & 76.81 & 76.07 & 76.12 & 76.10 & 76.83 & 76.60 \\
\hline 23 & DGD $\angle \mathrm{VP}$ & 102.96 & 103.19 & 103.93 & 103.88 & 103.90 & 103.17 & 103.39 \\
\hline 24 & DGD7 & 77.29 & 77.04 & 76.32 & 76.36 & 76.34 & 76.96 & 76.71 \\
\hline 24 & $D G D \angle V P 2$ & 102.70 & 102.96 & 103.68 & 103.64 & 103.66 & 103.04 & 103.28 \\
\hline & & 77.18 & 76.83 & 75.97 & 76.01 & 76.00 & 76.75 & 77.08 \\
\hline 25 & Det2/ZVP & 102.82 & 103.17 & 104.03 & 103.99 & 104.00 & 103.25 & 102.91 \\
\hline & & 79.94 & 79.59 & 78.96 & 78.99 & 78.97 & 79.35 & 78.57 \\
\hline 26 & LANL2DZ & 100.06 & 100.41 & 101.04 & 101.01 & 101.03 & 100.65 & 101.43 \\
\hline 27 & CEP-121G & 76.45 & 76.13 & 75.24 & 75.30 & 75.29 & 76.03 & 75.60 \\
\hline & & 103.55 & 103.87 & 104.76 & 104.70 & 104.71 & 103.97 & 104.39 \\
\hline & & 76.44 & 76.18 & 75.11 & 75.20 & 75.19 & 76.17 & 76.36 \\
\hline 28 & $T \angle \mathrm{VP}$ & 103.56 & 103.82 & 104.89 & 104.80 & 104.81 & 103.83 & 103.64 \\
\hline 29 & & 77.35 & 76.97 & 76.08 & 76.13 & 76.12 & 76.82 & 76.97 \\
\hline & Q $\angle \mathrm{VP}$ & 102.65 & 103.03 & 103.92 & 103.87 & 103.88 & 103.18 & 103.03 \\
\hline
\end{tabular}

The EA values of the $\mathrm{ZnO}$ dimer reported in Table 5 are positive. and are in the range of $0.27-2.43 \mathrm{eV}$ and comparatively lower than the monomer values.

The minimum energy needed to eject an electron, without changing the internuclear distances from the negative ion of a molecule in its ground state is defined as the vertical displacemnt energy VDE. The literature value of VDE for the $\mathrm{ZnO}$ monomer is $2.03 \mathrm{eV}(35,36)$.

Table 6 and 7 show the calculated values of VDE for both $\mathrm{ZnO}$ monomer and dimer. For the monomer the values are between $0.27-7.29 \mathrm{eV}$. Most of them are close to 2.00 $\mathrm{eV}$ including the B3LYP/DGDZVP2 level of theory (which is $2.16 \mathrm{eV})$. 
Table 4. Monomer EA/eV

\begin{tabular}{|c|c|c|c|c|c|c|c|c|}
\hline & \multirow{2}{*}{$\begin{array}{l}\text { Functional } \\
\text { Basis Set }\end{array}$} & \multicolumn{6}{|c|}{ DFT } & \multirow{2}{*}{$\operatorname{ccsD}(T)$} \\
\hline & & B3LYP & B3PW91 & PBEPBE & BVP86 & BP86 & M06 & \\
\hline 1 & $6-31 \mathrm{G}$ & 1.89 & 1.944 & 1.539 & 1.944 & 1.701 & 1.89 & 1.215 \\
\hline 2 & $6-31+G$ & 2.295 & 2.403 & 2.322 & 2.457 & 2.484 & 2.538 & 2.025 \\
\hline 3 & $6-31++G$ & 2.295 & 2.403 & 2.322 & 2.457 & 2.484 & 2.538 & 2.025 \\
\hline 4 & $6-311 G$ & 1.188 & 1.107 & 0.81 & 0.567 & 0.594 & 1.296 & - \\
\hline 5 & $6-311++G$ & 2.403 & 2.322 & 2.16 & 2.511 & 2.241 & 2.268 & 1.863 \\
\hline 6 & $6-31 G(d)$ & 1.728 & 1.782 & 1.647 & 1.782 & 1.809 & 1.728 & 1.269 \\
\hline 7 & $6-31 G(d, p)$ & 1.728 & 1.782 & 1.647 & 1.782 & 1.809 & 1.728 & 1.269 \\
\hline 8 & $6-31+G(d)$ & 2.268 & 2.376 & 2.295 & 2.457 & 2.187 & 2.268 & 1.863 \\
\hline 9 & $6-31+G(2 d)$ & 2.16 & 2.268 & 2.187 & 2.349 & 2.349 & 2.187 & 1.89 \\
\hline 10 & $6-31+G(2 d, p)$ & 2.16 & 2.268 & 2.187 & 2.349 & 2.349 & 2.187 & 1.89 \\
\hline 11 & $6-31+G(2 d f, p)$ & 2.43 & 2.241 & 2.16 & 2.295 & 2.322 & 2.43 & 1.836 \\
\hline 12 & $6-31+G(2 d f, 2 p)$ & 2.43 & 2.241 & 2.16 & 2.295 & 2.322 & 2.43 & 1.836 \\
\hline 13 & $6-31+G(3 d f, 2 p)$ & 2.403 & 2.214 & 2.133 & 2.295 & 2.322 & 2.403 & 2.079 \\
\hline 14 & $6-31++G(d)$ & 2.268 & 2.376 & 2.295 & 2.457 & 2.187 & 2.268 & 1.863 \\
\hline 15 & $6-31++G(d, p)$ & 2.268 & 2.376 & 2.295 & 2.457 & 2.187 & 2.268 & 1.863 \\
\hline 16 & $6-311+G(d)$ & 2.268 & 2.16 & 2.268 & 2.349 & 2.376 & 2.403 & 1.863 \\
\hline 17 & $6-311 G(d, p)$ & 1.053 & 0.945 & 0.648 & 0.945 & 0.702 & 1.431 & -0.081 \\
\hline 18 & $6-311++G(d)$ & 2.268 & - & 2.268 & 2.349 & 2.376 & 2.403 & 1.863 \\
\hline 19 & $6-311++G(d, p)$ & 2.268 & 2.16 & 2.268 & 2.349 & 2.376 & 2.403 & 1.863 \\
\hline 20 & cc-PVQZ & 2.268 & 2.187 & 2.079 & 2.16 & 2.187 & 2.052 & 1.809 \\
\hline 21 & aug-cc-PVDZ & 2.268 & 2.133 & 2.079 & 2.214 & 2.214 & 2.349 & -1.431 \\
\hline 22 & aug-cc-PVTZ & 2.16 & 2.349 & 2.268 & 2.376 & 2.376 & 2.214 & 2.025 \\
\hline 23 & DGDZVP & 1.917 & 1.836 & 1.62 & 1.701 & 1.701 & 1.809 & 1.296 \\
\hline 24 & DGDZVP2 & 2.025 & 1.971 & 1.728 & 1.782 & 1.809 & 1.917 & 1.323 \\
\hline 25 & Def2TZVP & 2.079 & 2.241 & 1.917 & 2.295 & 2.295 & 2.133 & 1.674 \\
\hline 26 & LANL2DZ & 1.836 & 2.025 & 1.728 & 2.025 & 1.782 & - & 1.404 \\
\hline 27 & CEP-121G & 2.025 & 2.079 & 1.917 & 2.052 & 2.241 & 2.133 & 1.485 \\
\hline 28 & TZVP & 1.89 & 1.755 & 1.485 & 1.836 & 1.593 & 1.89 & 1.107 \\
\hline 29 & QZVP & 2.322 & 2.214 & 2.106 & 2.214 & 2.241 & 2.268 & 1.809 \\
\hline
\end{tabular}

Table 5. Dimer Electron Affinity /eV

\begin{tabular}{|c|c|c|c|c|c|c|c|c|}
\hline & $\begin{array}{l}\text { Functional } \\
\text { Basis Set }\end{array}$ & B3LYP & B3PW91 & $\begin{array}{r}\text { DFT } \\
\text { PBEPBE }\end{array}$ & BVP86 & BP86 & M06 & $\operatorname{ccsD}(T)$ \\
\hline 1 & $3-21 G$ & 1.67 & 1.62 & 1.62 & 1.89 & 1.62 & 1.35 & 1.08 \\
\hline 2 & $6-31 G$ & 1.73 & 1.62 & 1.62 & 1.89 & 1.89 & 1.62 & 1.35 \\
\hline 3 & $6-31+G$ & 2.16 & 2.16 & 2.16 & 2.43 & 2.43 & 2.16 & 1.62 \\
\hline 4 & $6-31++G$ & 2.16 & 2.16 & 2.16 & 2.43 & 2.43 & 2.16 & 1.62 \\
\hline 5 & $6-311 G$ & 0.54 & 0.54 & 0.54 & 0.54 & 0.81 & 0.54 & - \\
\hline 6 & $6-311++G$ & 2.16 & 1.89 & 2.16 & 2.43 & 2.16 & - & - \\
\hline 7 & $6-31 G(d)$ & 1.62 & 1.62 & 1.62 & 1.62 & 1.62 & 1.62 & - \\
\hline 8 & $6-31 G(d, p)$ & 1.62 & 1.62 & 1.62 & 1.62 & 1.62 & 1.62 & - \\
\hline 9 & $6-31+G(d)$ & 2.16 & 2.16 & 1.89 & 2.43 & 2.16 & 2.16 & 1.62 \\
\hline 10 & $6-31+G(2 d)$ & 2.16 & 1.89 & 1.89 & 2.43 & 2.16 & 2.16 & 1.89 \\
\hline 11 & $6-31+G(2 d, p)$ & 2.16 & 1.89 & 1.89 & 2.43 & 2.16 & 2.16 & 1.89 \\
\hline 12 & $6-31+G(2 d f, p)$ & 2.16 & 2.16 & 1.89 & 2.16 & 2.16 & 2.16 & 1.89 \\
\hline 13 & $6-31+G(2 d f, 2 p)$ & 2.16 & 2.16 & 1.89 & 2.16 & 2.16 & 2.16 & 1.89 \\
\hline 14 & $6-31+G(3 d f, 2 p)$ & 1.89 & 1.89 & 2.16 & 2.16 & 2.16 & 2.16 & - \\
\hline 15 & $6-31++G(d)$ & 2.16 & 2.16 & 1.89 & 2.43 & 2.16 & 2.16 & 1.62 \\
\hline 16 & $6-31++G(d, p)$ & 2.16 & 2.16 & 1.89 & 2.43 & 2.16 & 2.16 & 1.62 \\
\hline 17 & $6-311+G(d)$ & 2.16 & 1.89 & 2.16 & 2.16 & 2.16 & 2.16 & 1.89 \\
\hline 18 & $6-311 G(d, p)$ & 0.54 & 0.54 & 0.27 & 0.54 & 0.54 & 0.27 & - \\
\hline 19 & $6-311++G(d)$ & 2.16 & 1.89 & 2.16 & 2.16 & 2.16 & 2.16 & 1.89 \\
\hline 20 & $6-311++G(d, p)$ & 2.16 & 1.89 & 2.16 & 2.16 & 2.16 & 2.16 & 1.89 \\
\hline 21 & aug-cc-PVDZ & 1.89 & 1.89 & 1.89 & 2.16 & 2.43 & 1.89 & - \\
\hline 22 & aug-cc-PVTZ & 2.16 & 1.89 & 2.16 & 2.16 & 2.16 & 1.89 & - \\
\hline 23 & DGDZVP & 1.62 & 1.62 & 1.62 & 1.89 & 1.89 & 1.35 & 1.35 \\
\hline 24 & DGDZVP2 & 1.62 & 1.62 & 1.62 & 1.89 & 1.89 & 1.89 & - \\
\hline 25 & Def2TZVP & 1.89 & 2.16 & 1.89 & 1.89 & 1.89 & 1.89 & - \\
\hline 26 & LANL2DZ & 1.89 & 1.89 & 1.62 & 1.89 & 1.89 & 1.89 & 1.62 \\
\hline 27 & CEP-121G & 2.16 & 1.89 & 2.16 & 2.16 & 1.89 & 1.89 & 1.62 \\
\hline 28 & TZVP & 1.62 & 1.35 & 1.62 & 1.62 & 1.62 & 1.62 & - \\
\hline 29 & QZVP & 1.89 & 1.89 & 2.16 & 2.16 & 2.16 & 1.89 & - \\
\hline
\end{tabular}

The VDE values of the following functionals and basis sets: 203 the B3LYP functional, and $6-31+\mathrm{G}(2 \mathrm{~d}), 6-31+\mathrm{G}(2 \mathrm{~d}, \mathrm{p})$, 204 aug-cc-PVTZ and Def2TZVP basis sets ; the B3PW91 func- 205 tional, and $6-31+\mathrm{G}(2 \mathrm{df}, \mathrm{p}), 6-31+\mathrm{G}(2 \mathrm{df}, 2 \mathrm{p}), 6-31+\mathrm{G}(3 \mathrm{df}, 2 \mathrm{p}), \quad 206$ $6-311+\mathrm{G}(\mathrm{d}), \quad 6-311++\mathrm{G}(\mathrm{d}, \mathrm{p}), \quad$ cc-PVQZ, aug-cc-PVDZ, $\quad{ }_{207}$ DGDZVP2,Def2TZVP,LANL2DZ,CEP-121G and QZVP basis sets ; the PBEPBE functional, and 6-311++G, 6-31+G(2d), 6$31+\mathrm{G}(2 \mathrm{~d}, \mathrm{p}), 6-31+\mathrm{G}(2 \mathrm{df}, \mathrm{p}), 6-31+\mathrm{G}(2 \mathrm{df}, 2 \mathrm{p}), 6-31+\mathrm{G}(3 \mathrm{df}, 2 \mathrm{p})$, cc-PVQZ, aug-cc-PVDZ and QZVP; BVP86 functional, and cc-PVQZ, aug-cc-PVDZ,LANL2DZ, CEP-121G and QZVP, BP86 functional, 6-31+G(2d), 6-31+G(2d,p), cc-PVQZ, DGDZVP2, Def2TZVP, CEP-121G and TZVP bais sets ; the M06 functional, and $6-31+\mathrm{G}(2 \mathrm{~d}), 6-31+\mathrm{G}(2 \mathrm{~d}, \mathrm{p})$, cc-PVQZ, DGDZVP2, Def2TZVP, CEP-121G and TZVP baisi sets ; the $\operatorname{CSSD}(\mathrm{T})$ method, with the $6-31+\mathrm{G}, 6-31++\mathrm{G}$, $6-31+\mathrm{G}(3 \mathrm{df}, 2 \mathrm{p})$ and aug-cc-PVTZ basis sets are $2.16 \mathrm{eV}$ with is close to the experimental value recorded within $\pm 0.13 \mathrm{eV}$. The VDE values for dimer are in the range of $0.27-2.43 \mathrm{eV}$. In the table 7 , only $\operatorname{CCDS}(\mathrm{T}) / 6-311 \mathrm{G}(\mathrm{d}, \mathrm{p})$ shows a negative VDE value.

Considering both the geometrical and electronic properties, we suggest that the combination of the B3LYP exchange functional and DB3LYPGDZVP2 basis set (B3LYP/DB3LYPGDZVP2) would be the most cost effective and reliable method to use in the study of $\mathrm{ZnO}$ clusters as catalysts for which the EA and VDE closely agree with the experimental values. 
Table 7. Dimer VDE/eV

\begin{tabular}{lllllllll} 
& Functional & \multicolumn{7}{c}{ DFT } \\
& Basis Set & B3LYP & B3PW91 & PBEPBE & BVP86 & BP86 & M06 & CCSD(T) \\
\hline 1 & 3-21G & 1.62 & 1.62 & 1.62 & 1.89 & 1.62 & 1.35 & - \\
2 & 6-31G & 1.89 & 1.62 & 1.62 & 1.89 & 1.89 & 1.62 & 1.35 \\
3 & 6-31+G & 2.16 & 2.16 & 2.43 & 2.43 & 2.43 & 2.16 & 1.89 \\
4 & 6-31++G & 2.16 & 2.16 & 2.43 & 2.43 & 2.43 & 2.16 & 1.89 \\
5 & 6-311G & 0.54 & 0.54 & 0.54 & 0.54 & 0.81 & 0.54 & - \\
6 & 6-311++G & 2.16 & 2.16 & 2.16 & 2.43 & 2.16 & - & - \\
7 & 6-31G(d) & 1.62 & 1.62 & 1.62 & 1.62 & 1.62 & 1.62 & - \\
8 & 6-31G(d,p) & 1.62 & 1.62 & 1.62 & 1.62 & 1.62 & 1.62 & - \\
9 & 6-31+G(d) & 2.16 & 2.16 & 1.89 & 2.43 & 2.16 & 2.16 & 1.62 \\
10 & 6-31+G(2d) & 2.16 & 1.89 & 1.89 & 2.43 & 2.16 & 2.16 & 1.89 \\
11 & 6-31+G(2d,p) & 2.16 & 1.89 & 1.89 & 2.43 & 2.16 & 2.16 & 1.89 \\
12 & 6-31+G(2df,p) & 2.16 & 2.16 & 1.89 & 2.43 & 2.16 & 2.16 & - \\
13 & 6-31+G(2df,2p) & 2.16 & 2.16 & 1.89 & 2.43 & 2.16 & 2.16 & - \\
14 & 6-31+G(3df,2p) & 2.16 & 1.89 & 2.16 & 2.16 & 2.16 & 2.16 & - \\
15 & 6-31++G(d) & 2.16 & 2.16 & 1.89 & 2.43 & 2.16 & 2.16 & 1.62 \\
16 & 6-31++G(d,p) & 2.16 & 2.16 & 1.89 & 2.43 & 2.16 & 2.16 & 1.62 \\
17 & 6-311+G(d) & 2.16 & 1.89 & 2.16 & 2.16 & 2.16 & 2.16 & 1.89 \\
18 & 6-311G(d,p) & 0.54 & 0.54 & 0.27 & 0.54 & 0.54 & 0.27 & -0.27 \\
19 & 6-311++G(d) & 2.16 & 1.89 & 2.16 & 2.16 & 2.16 & 2.16 & 1.89 \\
20 & 6-311++G(d,p) & 2.16 & 1.89 & 2.16 & 2.16 & 2.16 & 2.16 & 1.89 \\
21 & aug-cc-PVDZ & 1.89 & 2.16 & 1.89 & 2.43 & 2.43 & 2.16 & - \\
22 & aug-cc-PVTZ & 2.16 & 1.89 & 2.16 & 2.16 & 2.16 & 2.16 & - \\
23 & DGDZVP & 1.62 & 1.62 & 1.62 & 1.89 & 1.89 & 1.35 & 1.35 \\
24 & DGDZVP2 & 1.62 & 1.62 & 1.62 & 1.89 & 1.89 & 1.89 & - \\
25 & Def2TZVP & 1.89 & 2.16 & 1.89 & 1.89 & 2.16 & 2.16 & - \\
26 & LANL2DZ & 1.89 & 1.89 & 1.62 & 1.89 & 1.89 & 1.89 & 1.62 \\
27 & CEP-121G & 2.16 & 1.89 & 2.16 & 2.16 & 2.16 & 2.16 & 1.62 \\
\hline & & & & & & & &
\end{tabular}

After choosing suitable basis sets for our study of the properties of $\mathrm{ZnO}$, we also investigated suitable cluster sizes to study photocatalysis. Our primary goal was to identify a reliable photocatalyst from amongst $\mathrm{ZnO}$ clusters to produce $\mathrm{H}_{2}$ and $\mathrm{O}_{2}$ by splitting two water molecules.

The energy gap between HOMO and LUMO is one of the important parameters that identify cluster stability. We compare the HOMO-LUMO gap of small $\mathrm{ZnO}$ clusters, where the cluster size is from 1-6 in Table 8 using the B3LYP functional and 6-311++G, DGDZVP, DGDZVP2 and aug-cc-PVDZ basis sets. Results using B3LYP/DGDZVP2 level of theory are shown in the Figure 2 and other results are shown in the SI document.

Cluster sizes 4 and 5 have relatively higher HOMOLUMO gaps (4.66 eV and $4.79 \mathrm{eV}$ respectively using B3LYP/DGDZVP2 method) indicating that they would be relatively less efficient than the other neutral clusters. The $(\mathrm{ZnO})_{3}$ and $(\mathrm{ZnO})_{6}$ have lower HOMO-LUMO energy gaps(4.42 eV and $3.76 \mathrm{eV}$ using the same method) compared to the $4^{\text {th }}$ and $5^{\text {th }}$ clusters, and indicate that the $3^{\text {th }}$ and $6^{\text {th }} \mathrm{ZnO}$ clusters have comparably higher reactivity. When considering the photocatalytic water splitting reaction to produce $\mathrm{H}_{2}$, the minimum photon energy required for the overall reaction is $1.23 \mathrm{eV}$ which indicates that all the small $\mathrm{ZnO}$ nanoclusters studied are possible candidates to catalyze the photosplitting of water.
Table 8. HOMO-LUMO gap in eV

\begin{tabular}{lllll} 
Cluster Size & \multicolumn{4}{c}{ Basis set } \\
& $6-311++G$ & DGDZVP & DGDZVP2 & aug-cc-PVDZ \\
\hline 1 & 2.29 & 2.33 & 2.35 & 2.36 \\
2 & 2.52 & 2.58 & 2.63 & 2.69 \\
3 & 4.17 & 4.36 & 4.42 & 4.35 \\
4 & 4.39 & 4.59 & 4.66 & 4.56 \\
5 & 4.56 & 4.72 & 4.79 & 4.69 \\
6 & 3.52 & 3.71 & 3.76 & 3.68 \\
\hline
\end{tabular}

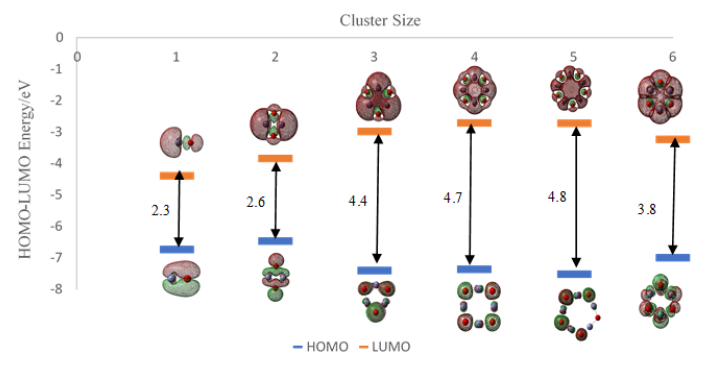

Fig. 2. HOMO-LUMO gap of $(\mathrm{ZnO}) \mathrm{n}, \mathrm{n}=1-6$ using B3LYP/DGDZVP2. Images use the coarse grid method and isoval $=0.02$.

We have also calculated the vibrational frequencies in order to check the stability of the optimized geometries. Infrared spectra for $(\mathrm{ZnO})_{\mathrm{n}} ; \mathrm{n}=1-6$ are shown in Figure 3 and all the relevant frequency values for the monomer, dimer and their anions are tabulated in the SI document. There were no imaginary vibrational frequencies in our study of any of the clusters. The monomer $\mathrm{ZnO}$ has one wide peak at $708 \mathrm{~cm}^{-1}$. The dimer $\mathrm{ZnO}$ has three major peaks at $213 \mathrm{~cm}^{-1}, 441 \mathrm{~cm}^{-1}$ and $566 \mathrm{~cm}^{-1}$ frequencies. The $(\mathrm{ZnO})_{3}$ has a major peak at $648 \mathrm{~cm}^{-1}$ and a minor peak at $221 \mathrm{~cm}^{-1},(\mathrm{ZnO})_{4}$ has a major peak at $735 \mathrm{~cm}^{-1}$ and two minor peaks at $176 \mathrm{~cm}^{-1}$ and 225 $\mathrm{cm}^{-1},(\mathrm{ZnO})_{5}$ has one major peak at $801 \mathrm{~cm}^{-1}$ and 3 minor peaks at $174 \mathrm{~cm}^{-1}, 221 \mathrm{~cm}^{-1}$ and $518 \mathrm{~cm}^{-1}$ and the $(\mathrm{ZnO})_{6}$ two major peaks at $385 \mathrm{~cm}^{-1}$ and $581 \mathrm{~cm}^{-1}$ and a minor peak at $491 \mathrm{~cm}^{-1}$ frequencies.

The optimized energies of the singlet and the first excited triplet state of $(\mathrm{ZnO})_{n}, \mathrm{n}=1-6$ are calculated using the selected methods. The details are shown in the SI document. The energy difference of the singlet and the first excited triplet state are displayed in the table 9 . 


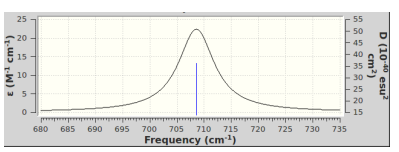

(a) $\mathrm{ZnO}$

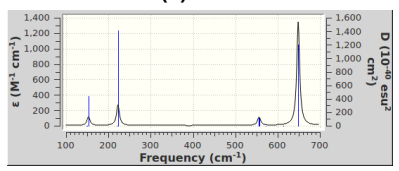

(c) $(\mathrm{ZnO})_{3}$

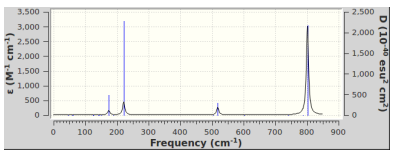

(e) $(\mathrm{ZnO})_{5}$

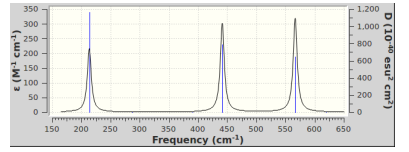

(b) $(\mathrm{ZnO})_{2}$

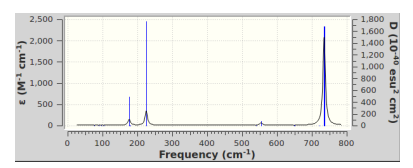

(d) $(\mathrm{ZnO})_{4}$

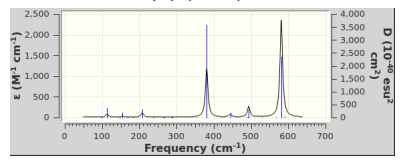

(f) $(\mathrm{ZnO})_{6}$
Fig. 3. Calculated infrared spectra for $(\mathrm{ZnO})_{n}$ structures $(a) n=1,(b) n=2,(c) n=3$, (d) $n=4,(e) n=5$ and (f) $n=6$.

$\mathrm{H}_{2}$ production in the water splitting reaction is an endothermic reaction that requires two $\mathrm{H}_{2} \mathrm{O}$ molecules. The heat of formation of $\mathrm{H}_{2} \mathrm{O}$ is $-57.8 \pm 0.02 \mathrm{kCal} / \mathrm{mol}$ at $298 \mathrm{~K}$ temperature, which is approximately comparable to the energy of two photons at $500 \mathrm{~nm}(38)$.

Table 9. Singlet-Triplet energy difference in $\mathrm{k} \mathrm{cal} / \mathrm{mol}$ at B3LYP functional

\begin{tabular}{llllllll} 
& Basis set & \multicolumn{7}{c}{ Cluster Size (n) } \\
& & 1 & 2 & 3 & 4 & 5 & 6 \\
\hline 1 & $3-21 G$ & 9.78 & 7.17 & 55.34 & 71.95 & 76.64 & 58.15 \\
2 & $6-31+G$ & -3.73 & 13.14 & 52.82 & 63.63 & 68.71 & 54.67 \\
3 & $6-31++G$ & -3.73 & 13.14 & 52.82 & 63.63 & 68.71 & 54.80 \\
4 & $6-311 G$ & -14.36 & 6.01 & 53.50 & 54.75 & 62.44 & 53.13 \\
5 & 6-311++G & -3.87 & 13.65 & 53.02 & 63.90 & 69.05 & 54.98 \\
6 & 6-31G(d) & 2.12 & 17.70 & 59.70 & 69.41 & 77.65 & 61.57 \\
7 & $6-31+G(d)$ & 36.29 & 17.02 & 34.36 & 69.09 & 74.56 & 59.23 \\
8 & 6-31+G(2d) & 36.35 & 18.34 & 58.44 & 69.59 & 74.70 & 58.96 \\
9 & 6-31+G(2d,p) & 36.35 & 18.34 & 58.44 & 69.59 & 74.70 & 58.96 \\
10 & 6-31+G(2df,p) & 36.09 & 18.08 & 58.27 & 69.46 & 74.59 & 58.88 \\
11 & 6-31+G(2df,2p) & 36.09 & 18.08 & 58.27 & 69.46 & 74.59 & 58.88 \\
12 & 6-31+G(3df,2p) & 36.11 & 18.68 & 58.95 & 69.83 & 75.18 & 59.61 \\
13 & 6-31++G(d) & 36.29 & 17.02 & 57.55 & 69.09 & 74.56 & 59.23 \\
14 & 6-31++G(d,p) & 36.29 & 17.02 & 57.55 & 69.09 & 74.56 & 59.23 \\
15 & Aug-ccPVDZ & 35.21 & 18.55 & 58.52 & 69.73 & 74.84 & 58.90 \\
16 & Aug-ccPVTZ & 34.90 & 18.26 & 58.26 & 69.67 & 74.87 & 58.98 \\
17 & DGDZVP & 34.49 & 14.74 & 57.33 & 69.45 & 74.77 & 58.77 \\
18 & DGDZVP2 & 34.85 & 16.05 & 58.66 & 73.22 & 76.39 & 59.93 \\
19 & Def2TZVP & 34.30 & 16.48 & 57.65 & 69.17 & 74.48 & 58.32 \\
20 & TZVP & 33.48 & 15.29 & 56.12 & 67.04 & 72.00 & 57.47 \\
\hline & & & & & & &
\end{tabular}

$\mathrm{ZnO}$ clusters, $\mathrm{n}=1$ and 2 have values lower than than 57.8 $\mathrm{kCal} / \mathrm{mol}$ while $\mathrm{n}=4$ and 5 have higher values. Cluster size 3 and 6 have relatively values closer to the energy of 2 visible photons which would be desired cluster systems to study the photocatalytic water splitting reaction to produce $\mathrm{H}_{2}$ and $\mathrm{O}_{2}$ using 2 water molecules in the presence of $\mathrm{ZnO}$ nanolcusters as the catalyst.

\section{Conclusion}

We have investigated the structural and electronic properties of $\mathrm{ZnO}$ monomer and dimer and vibrational frequencies of both structures to choose suitable basis sets and exchange functionals to study photocatalytic effects of $\mathrm{ZnO}$ nanoclusters on water splitting. We also studied the singlet-triplet energy difference and HOMO-LUMO gap of $(\mathrm{ZnO})_{\mathrm{n}} ; \mathrm{n}=1-6$ to select the best cluster size of $\mathrm{ZnO}$ as a photocatalyst. By comparing our calculated values with experimental values, we find that the B3LYP/DGDZVP2 combination of exchange functional and basis set would be reliable and optimal in calculating the properties of $\mathrm{ZnO}$ nanoclusters using DFT with a relatively low computational time compared to other methods (e.g. CCSD $(\mathrm{T})$ ). From the singlet -triplet energy comparisons, both $n=3$ and $n=6$ nanocluster systems are also possible photocatalysts to use in the formation of $\mathrm{H}_{2}$ and $\mathrm{O}_{2}$ in a water splitting reaction, and we suggest that the $(\mathrm{ZnO})_{3}$ rather than the $(\mathrm{ZnO})_{6}$ is the better of the two photocatalysts considering the complexity of the reaction.

\section{Author Affiliations}

ORCID

Duwage Charitha Perera 0000-0003-4237-138X

Jayendran C. Rasaiah 0000-0002-4453-7438

\section{Corresponding Author}

Email: rasaiah@maine.edu

Notes

The authors declare no competing financial interest.

\section{Acknowledgment}

We thank Stephen Cousins, Bruce Segee and staff of the University of Maine High Performance Computing Group for their technical assistance and allotment of computer time.We also thank Eric Lovejoy for his assistance.

\section{Supporting Information Appendix (SI)}

The Supporting Information (SI) document is available for free of charge.

\section{References}

1. SA Claridge, et al., Cluster-Assembled Materials. ACS Nano 3, 244-255 (2009) Publisher: American Chemical Society.

2. P Jena, AW Castleman, Clusters: A bridge across the disciplines of physics and chemistry. Proc. Natl. Acad. Sci. 103, 10560-10569 (2006) Publisher: National Academy of Sciences Section: Introductory Perspective.

3. HG Byun, I Kim, HS Kwon, GT Bae, Comparisons of the Functional and Basis Set Combinations for Silicon Oxide Clusters: A Density Functional Theory Study: Comparisons of the Functional and Basis Set Combinations. Bull. Korean Chem. Soc. 38, 1310-1315 (2017).

4. S Li, DA Dixon, Benchmark Calculations on the Electron Detachment Energies of MO3- and M2O6- (M = Cr, Mo, W). The J. Phys. Chem. A 111, 11908-11921 (2007) Publisher: American Chemical Society. 
5. GT Bae, B Dellinger, RW Hall, Density Functional Calculation of the Structure and Electronic Properties of CunOn $(n=18)$ Clusters. The J. Phys. Chem. A 115, 2087-2095 (2011) Publisher: American Chemical Society.

6. L Guo, YL Ji, H Xu, P Simon, Z Wu, Regularly Shaped, Single-Crystalline ZnO Nanorods with Wurtzite Structure. J. Am. Chem. Soc. 124, 14864-14865 (2002) Publisher: American Chemical Society.

7. $\mathrm{X}$ Jiaqiang, $\mathrm{C}$ Yuping, $\mathrm{L}$ Yadong, $\mathrm{S}$ Jianian, Gas sensing properties of $\mathrm{ZnO}$ nanorods prepared by hydrothermal method. J. Mater. Sci. 40, 2919-2921 (2005).

8. Y Gong, M Zhou, L Andrews, Spectroscopic and Theoretical Studies of Transition Metal Oxides and Dioxygen Complexes. Chem. Rev. 109, 6765-6808 (2009) Publisher: American Chemical Society.

9. HJ Zhai, LM Wang, SD Li, LS Wang, Vibrationally Resolved Photoelectron Spectroscopy of BO- and BO2-: A Joint Experimental and Theoretical Study. The J. Phys. Chem. A 111, 1030-1035 (2007) Publisher: American Chemical Society.

10. AK Patra, A Dutta, A Bhaumik, Self-assembled ultra small $\mathrm{ZnO}$ nanocrystals for dyesensitized solar cell application. J. Solid State Chem. 215, 135-142 (2014).

11. C Clementi, et al., Photoluminescence properties of zinc oxide in paints: a study of the effect of self-absorption and passivation. Appl. Spectrosc. 66, 1233-1241 (2012).

12. SH Jin, et al., Water-Soluble Thin Film Transistors and Circuits Based on Amorphous Indium-Gallium-Zinc Oxide. ACS Appl. Mater. \& Interfaces 7, 8268-8274 (2015) Publisher: American Chemical Society.

13. ZL Wang, Zinc oxide nanostructures: growth, properties and applications. J. Physics: Condens. Matter 16, R829-R858 (2004) Publisher: IOP Publishing.

14. A Sirelkhatim, et al., Review on Zinc Oxide Nanoparticles: Antibacterial Activity and Toxicity Mechanism. Nano-Micro Lett. 7, 219-242 (2015).

15. SB Woodley, AA Sokol, CRA Catlow, AA AI-Sunaidi, SM Woodley, Structural and Optical Properties of $\mathrm{Mg}$ and $\mathrm{Cd}$ Doped ZnO Nanoclusters. The J. Phys. Chem. C 117, 2712727145 (2013).

16. MJS Spencer, Gas sensing applications of 1D-nanostructured zinc oxide: Insights from density functional theory calculations. Prog. Mater. Sci. 57, 437-486 (2012).

17. $\mathrm{J} \mathrm{Xu}$, et al., Uniform $\mathrm{ZnO}$ nanorods can be used to improve the response of $\mathrm{ZnO}$ gas sensor. Mater. Sci. Eng. B 150, 55-60 (2008).

18. SR Kelly, et al., ZnO As an Active and Selective Catalyst for Electrochemical Water Oxidation to Hydrogen Peroxide. ACS Catal. 9, 4593-4599 (2019) Publisher: American Chemical Society.

19. MA Johar, RA Afzal, AA Alazba, U Manzoor, Photocatalysis and Bandgap Engineering Using ZnO Nanocomposites. Adv. Mater. Sci. Eng. 2015, e934587 (2015) Publisher: Hindawi.

20. E Berardo, et al., Benchmarking the fundamental electronic properties of small tio 2 nanoclusters by gw and coupled cluster theory calculations. J. Chem. Theory Comput. 13, 3814-28 (2017).

21. X Chen, Z Wu, D Liu, Z Gao, Preparation of ZnO Photocatalyst for the Efficient and Rapid Photocatalytic Degradation of Azo Dyes. Nanoscale Res. Lett. 12, 143 (2017).

22. MM Brewster, $X$ Zhou, MY Lu, $S$ Gradečak, The interplay of structural and optical properties in individual ZnO nanostructures. Nanoscale 4, 1455-1462 (2012) Publisher: The Royal Society of Chemistry.

23. G Malloci, L Chiodo, A Rubio, A Mattoni, Structural and Optoelectronic Properties of Unsaturated $\mathrm{ZnO}$ and $\mathrm{ZnS}$ Nanoclusters. The J. Phys. Chem. C 116, 8741-8746 (2012) Publisher: American Chemical Society.

24. V Srikant, DR Clarke, On the optical band gap of zinc oxide. J. Appl. Phys. 83, 5447-5451 (1998) Publisher: American Institute of Physics.

25. SH Na, CH Park, First-Principles Study of the Surface of Wurtzite $\mathrm{ZnO}$ and $\mathrm{ZnS}$ - Implications for Nanostructure Formation. J. Korean Phys. Soc. 54, 867-872 (2009) Publisher: The Korean Physical Society.

26. L Wang, M Muhammed, Synthesis of zinc oxide nanoparticles with controlled morphology. J. Mater. Chem. 9, 2871-2878 (1999) Publisher: The Royal Society of Chemistry.

27. R Bovhyra, D Popovych, O Bovgyra, A Serednytski, Ab Initio Study of Structural and Electronic Properties of $(\mathrm{ZnO}) \mathrm{n}$ "Magical" Nanoclusters $\mathrm{n}=(34,60)$. Nanoscale Res. Lett. 12, 76 (2017).

28. AA Al-Sunaidi, AA Sokol, CRA Catlow, SM Woodley, Structures of Zinc Oxide Nanoclusters: As Found by Revolutionary Algorithm Techniques. The J. Phys. Chem. C 112, 18860-18875 (2008) Publisher: American Chemical Society.

29. CE Szakacs, EF Merschrod S., KM Poduska, Structural Features That Stabilize ZnO Clusters: An Electronic Structure Approach. Computation 1, 16-26 (2013).

30. M Chen, TP Straatsma, Z Fang, DA Dixon, Structural and Electronic Property Study of $(\mathrm{ZnO}) \mathrm{n}, \mathrm{n}$ 168: Transition from Zinc Oxide Molecular Clusters to Ultrasmall Nanoparticles. The J. Phys. Chem. C 120, 20400-20418 (2016) Publisher: American Chemical Society.

31. KDD Gunaratne, C Berkdemir, CL Harmon, AW Castleman, Investigating the Relative Stabilities and Electronic Properties of Small Zinc Oxide Clusters. The J. Phys. Chem. A 116, 12429-12437 (2012) Publisher: American Chemical Society.

32. MJ Frisch, et al., Gaussian 16 Revision C.01 (2016) Gaussian Inc. Wallingford CT.

33. R Dennington, TA Keith, JM Millam, Gaussview Version 6 (2019) Semichem Inc. Shawnee Mission KS.

34. J Matxain, A Irigoras Balda, J Fowler, J Ugalde, Electronic excitation energies of $\mathrm{Zn}$ i S i clusters. Phys. Rev. A 64, 013201 (2001).

35. CA Fancher, HL de Clercq, OC Thomas, DW Robinson, KH Bowen, Zinc oxide and its anion: A negative ion photoelectron spectroscopic study. The J. Chem. Phys. 109, 8426-8429 (1998).

36. JH Kim, et al., Vibrationally Resolved Photoelectron Spectroscopy of MgO- and ZnO- and the Low-Lying Electronic States of MgO, MgO-, and ZnO. The J. Phys. Chem. A 105, 5709-5718 (2001) Publisher: American Chemical Society.

37. L Christophorou, Atomic and molecular radiation physics, Wiley monographs in chemical physics. No. 0471156299, (year?)

38. Z Fang, DA Dixon, Computational Study of $\mathrm{H} 2$ and $\mathrm{O} 2$ Production from Water Splitting by Small (MO2)n Clusters (M = Ti, Zr, Hf). The J. Phys. Chem. A 117, 3539-3555 (2013) 